\title{
Optimal control of peritoneal dialysis
}

\section{Paolo Zunino*}

MOX, Politecnico di Milano

E-mail: paolo.zunino@nate.polimi.it

\section{Diego Mastalli ${ }^{\dagger}$}

EPFL, Lausanne

E-mail: diego.mastallicepfl.ch

For patients with end-stage renal failure, peritoneal dialysis (PD) is a well established therapeutic option among the medical therapies to purify blood and to extract fluid from patients. However, a high drop-out rate related to the progressive decrease in PD efficiency persists. New tools that would allow to optimize the present PD modalities and/or extend the therapeutic techniques are therefore welcomed. In this work, we set up an optimal control strategy to optimize PD.

Since a PD therapy can be regarded as a sequence of basic cycles, we focus on an optimal control problem governed by ordinary differential equations with emphasis on switched systems. We aim to approximate the gradient of a given cost functional with respect to the switching sequence that determine the therapeutic cycles and to apply an augmented Lagrangian algorithm to maximize the cost functional under constraints. Finally, numerical results and their application to PD are discussed.

Control Systems: Theory, Numerics and Applications

30 March - 1 April 2005

Rome

\footnotetext{
* Speaker.

${ }^{\dagger}$ This work was supported by Project N. 6169.2 of KTI/CTI, the Swiss Innovation Promotion Agency.
} 


\section{Problem set up}

The peritoneal dialysis is a therapy to purify blood and to extract fluid from patients with limited renal functions. Blood purification is obtained by exchanges of chemicals between blood and a dialyzing solution injected in the peritoneal cavity, namely the interstices of the viscera. Roughly speaking, the exchanges of chemicals take place across the membrane that lines the walls of the abdominal cavity and encloses the viscera, called peritoneum or peritoneal membrane.

Many works have been published in medical literature to describe exchanges of chemicals during PD with mathematical equations, for example we mention $[6,12,13,15]$. The models that are proposed are actually very similar, indeed they come from equations describing exchanges of many chemical species across a membrane separating two solutions with different concentrations. The application of the mass conservation principle allows to describe the evolution in time of the chemical species into account by means of a system of ordinary differential equations which represents the state equation for our optimization strategy.

\subsection{A mathematical model for PD}

During PD therapy, the exchange of chemicals takes place through the net of capillaries within the folded peritoneal membrane. For this reason the geometrical modeling of the domain to account for spatial variations would be extremely difficult. A space lumped model, in which the variations in space are neglected, looks therefore more suitable to study the kinetics of chemicals during the therapy (see figure 1 for a synoptic description of the model).

Our model exhibits one compartment accounting for the body, denoted by the index (b), and one for the peritoneal cavity of the patient, (d), that are separated by a semipermeable membrane that represents the peritoneal membrane. The latter compartment is filled by a solution of $m$ chemicals, denoted by the indices $i=1,2, \ldots, m$. Assuming that the concentrations are uniform in space, the physical quantities of interest are the volume of the solution and the total amount of each solute in the two compartments, namely $V_{b}, V_{d}, V_{b} c_{b, i}, V_{d} c_{b, i}$, where $c_{b, i}, c_{d, i}$ are the concentrations (mass of solute per volume of solution). The interaction between the two compartments is governed by the equations prescribing the flux of dialyzing fluid, $J_{v}$, and of chemical species, $J_{s, i}$, across the membrane. A well accepted mathematical model for these fluxes is due to Kedem and Katchalsky $[7,8]$. In this model the membrane is characterized by a set of pores that allow the exchange of fluid and of chemical species between the two compartments. The pores can be subdivided in different classes that we denote by the index $j=1, \ldots, p$, depending on their size. Then, we introduce $L_{p}, P_{i}$, the hydraulic conductivity and permeability of the membrane. Let us denote with $L_{p, j}, P_{i, j}$ the corresponding quantities associated to the $j^{\text {th }}$ class of pores. Furthermore let $s_{i, j}$ be the sieving coefficients of the membrane relative to $j^{t h}$ class of pores with respect to the $i^{\text {th }}$ molecule. However, in what follows we will use the reflection coefficients $\sigma_{i, j}=1-s_{i, j}$ (the complementary of $s_{i, j}$ with respect to the unity), which determine the ratio of molecules that can sieve across the membrane. The Kedem-Katchalsky equations read as follows,

$$
\begin{gathered}
J_{v, j}=L_{p, j}\left([p]-\sum_{i=1, m} \sigma_{i, j}\left[\pi_{i}\right]\right), \quad J_{v}=\sum_{j=1, p} J_{v, j} \\
J_{s, i, j}=P_{i, j}\left[c_{i}\right]+J_{v, j}\left(1-\sigma_{i, j}\right)\left\langle c_{b, i}, c_{d, i}\right\rangle, \quad J_{s, i}=\sum_{j=1, p} J_{s, i, j},
\end{gathered}
$$


where, in this context, $\left\langle c_{b, i}, c_{d, i}\right\rangle$ is the average concentration inside the membrane and $[c]=$ $c_{b}-c_{d},[p]=p_{b}-p_{d}$, while $\pi_{i}=R T c_{i}$, being $R$ and $T$ be the gas constant and the absolute temperature, according to Van't Hoff law, [3]. By plugging the definitions of $J_{v}$ and $J_{s, i}$ into the mass conservation principle, we end up with a system of $M=2 m+2$ ordinary differential equations that describe the rate of change of the unknowns $V_{b}, V_{d}, V_{b} c_{b, i}, V_{d} c_{d, i}, i=1, \ldots, m$ by means of expressions derived from equations (1.1) and (1.2). If we denote the rate of change of the variables at hand with $\left.d_{t} V_{b}, d_{t} V_{d}, d_{t} V_{b} c_{b, i}, d_{t} V_{d} c_{d, i}\right)$ we have,

$$
\left\{\begin{array}{l}
d_{t} V_{b}=-\sum_{j=1, p}\left\{L_{p, j} \cdot S \cdot\left([p]-R T \sum_{i=1, m} \sigma_{i, j}\left[c_{i}\right]\right)\right\}+J v_{l y m p h} \\
d_{t} V_{d}=\sum_{j=1, p}\left\{L_{p, j} \cdot S \cdot\left([p]-R T \sum_{i=1, m} \sigma_{i, j}\left[c_{i}\right]\right)\right\}+u(t)-J v_{l y m p h} \\
d_{t}\left(V_{b} c_{b, i}\right)=-\sum_{j=1, p}\left\{P_{i, j}\left[c_{i}\right]+J_{v, j}\left(1-\sigma_{i, j}\right)\left\langle c_{b, i}, c_{d, i}\right\rangle\right\} \cdot S+q_{i}+K r_{i}, i=1, \ldots, m \\
d_{t}\left(V_{d} c_{d, i}\right)=\sum_{j=1, p}\left\{P_{i, j}\left[c_{i}\right]+J_{v, j}\left(1-\sigma_{i, j}\right)\left\langle c_{b, i}, c_{d, i}\right\rangle\right\} \cdot S, \quad i=1, \ldots, m
\end{array}\right.
$$

where $S$ represents the effective surface of the peritoneal membrane, $J v_{\text {lymph }}$ takes into account the drainage due to the lymphatic system, $q_{i}$ represents the generation rate of the $i^{\text {th }}$ molecule inside the body, $K r_{i}$ represents the residual renal function and $u(t)$ is a source term that takes into account of the volume of fluid that is periodically injected and extracted from the peritoneal cavity by the pump. The system of equations (1.3) is the state equation of our optimal control problem and the function $u(t)$ is the control. Before proceeding, we introduce an abridged notation for the state equation. We denote with $\mathbf{x}=\left[V_{b}, c_{b, i}, V_{d}, c_{d, i}\right] \in \mathbb{R}^{M}$, and with $\mathbf{f}(\mathbf{x}, u) \in \mathbb{R}^{M}$ the right hand side of (1.3). Furthermore, the derivative with respect to time of a variable $v(t)$ will be represented concisely with $\dot{v}=d_{t} v$. As a result of that, the state equation will be thus rewritten as $\dot{\mathbf{x}}=\mathbf{f}(\mathbf{x}, u)$ and its well posedness will be discussed later on.

\subsection{Definition of the control $u(t)$}

Blood purification in PD is obtained by exchanges of chemicals between blood and a solution injected in the peritoneal cavity, called dialyzing fluid. The solution in the peritoneal cavity is periodically replaced by injecting or extracting the dialyzing fluid from the patient, through an external pump (figure 1 shows a simplified scheme of the process). The idea that lays at the basis of this work is thus to devise a procedure to find for each patient the profile of injections and extractions that ensures the best blood purification.

The amount of fluid injected or extracted from the peritoneal cavity in a given time is determined by the pump flow rate $u(t)$. We observe that the function $u(t)$ is a piecewise constant function that can assume values in the set $\{U, 0,-U\}$ corresponding to the injection, dwell (the part of the PD cycle when the dialyzing fluid rests into the peritoneal cavity) and extraction modes, being $U$ the nominal flow rate of the pump. Indeed, the degrees of freedom of our control are the instants when the pump switches to one mode to the other. In order to define $u(t)$, we introduce a suitable partition of the time interval corresponding to the duration of the therapy. This partition represents the subdivision of the therapy in cycles and each cycle then subdivided in subintervals 


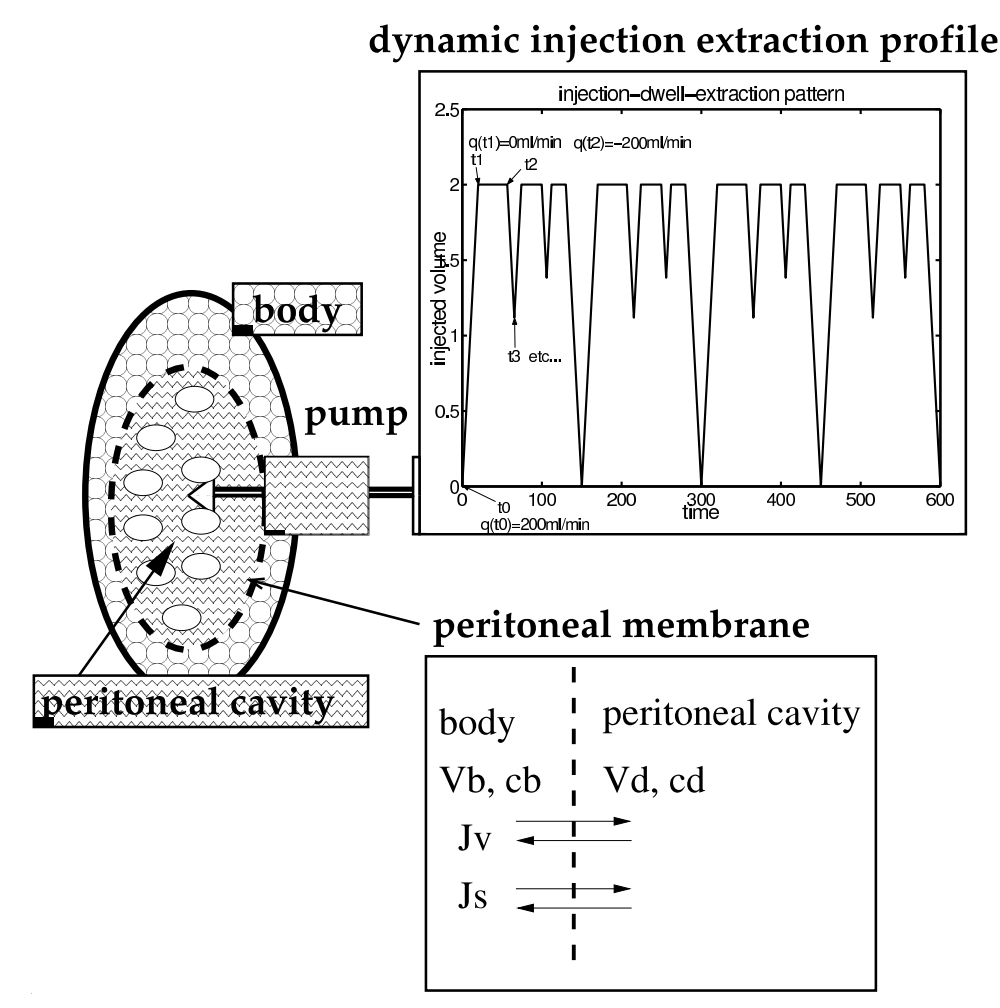

Figure 1: A synoptic description of peritoneal dialysis

corresponding to injection, dwell and extraction. Let us denote with $N$ the number of cycles associated to the therapy, then we introduce $I_{i}=\left[T_{i}, T_{i+1}\right]$, with $i=1, \cdots, N$, the interval associated to each injection-dwell-extraction cycle and let be $T_{N+1}=T_{1}+T_{t o t}$, being $T_{t o t}$ the total duration of the therapy. Each cycle should be further subdivided in the dwell phase (characterized by $u(t)=0$ for $t \in\left[T_{i}, t_{i}^{\prime}\right]$ where $t_{i}^{\prime}$ is the switching instant between dwell and extraction), the extraction phase (characterized by $u(t)=-U$ for $t \in\left[t_{i}^{\prime}, t_{i}^{\prime \prime}\right]$ where $t_{i}^{\prime \prime}$ is the switching instant between extraction and injection) and the injection phase (characterized by $u(t)=U$ for $t \in\left[t_{i}^{\prime \prime}, t_{i}^{\prime \prime \prime}=T_{i+1}\right]$ where $t_{i}^{\prime \prime \prime}$ is the switching instant between injection and dwell). Finally, any admissible control $u(t)$ should satisfy the following constraints:

(a) the maximal duration of the therapy is fixed $T_{N+1}-T_{1}=T_{\text {tot }}$

(b) the dialyzing fluid in the peritoneal cavity should not exceed the maximal capacity, denoted with $V_{\max }$ :

$$
U\left(t_{i}^{\prime \prime \prime}-t_{i}^{\prime \prime}\right) \leq V_{\max }
$$

(c) the maximal amount of dialyzing fluid, denoted with $V_{\text {tot }}$ can not be exceeded:

$$
U \sum_{i=1}^{N}\left(t_{i}^{\prime \prime \prime}-t_{i}^{\prime \prime}\right)=V_{t o t}
$$

(d) during the dwell phase the peritoneal cavity should be completely filled: 


$$
U\left(t_{i}^{\prime \prime}-t_{i}^{\prime}\right)=U\left(t_{i}^{\prime \prime \prime}-t_{i}^{\prime \prime}\right)
$$

This constraint allows to eliminate $N$ degrees of freedom because it determines

$$
t_{i}^{\prime \prime}=\frac{t_{i}^{\prime \prime \prime}+t_{i}^{\prime}}{2}
$$

It is clear that the function $u(t)$ can be uniquely identified by fixing a finite number of degrees of freedom that correspond to the switching instants $\mathbf{t}=\left[T_{i}, t_{i}^{\prime}\right]_{i=1}^{N}$. The number of degrees of freedom (dof) of $\mathbf{t}$ is thus dof $=2(N-1)$, since the initial and final times $T_{1}$ and $T_{N+1}$ are fixed. For the sake of simplicity, we will renumber the degrees of freedom as follows, $\mathbf{t}=\left[t_{i}\right]_{i=1}^{\text {dof }}$. We finally observe that the inequality and equality constraints, (1.4) and (1.5) respectively, can be reformulated for the vector of unknowns t. More precisely, equation (1.4) becomes,

$$
g_{i}(\mathbf{t}) \leq 0, i=1, \ldots, N-1, \quad \text { with } \quad g_{i}(\mathbf{t})=U\left(t_{2 i+1}-\frac{t_{2 i}+t_{2 i+1}}{2}\right)-V_{\max }
$$

while equation (1.5) becomes,

$$
h(\mathbf{t})=0, \quad \text { with } \quad h(\mathbf{t})=U \sum_{i=1}^{N-1}\left(t_{2 i+1}-\frac{t_{2 i}+t_{2 i+1}}{2}\right)-V_{t o t}=0 .
$$

\subsection{The control problem}

Before setting up our control problem, we observe that, since the control $u(t)$ is a discontinuous function, the solution of the state equation is not defined in the classical sense. As a consequence of that, we split the state equation on the subdomains $I_{i}$ where the function $u(t)$ is continuous and in particular it is constant. More precisely, our state problem becomes,

Problem 1. Given $\mathbf{x}_{0}$, find $\mathbf{x}(t) \in\left(C^{0}\left(\left[T_{1}, T_{N+1}\right]\right)\right)^{M}$ such that,

$$
\left\{\begin{array}{l}
\dot{\mathbf{x}}(t)=\mathbf{f}(\mathbf{x}(t), u(t)), \quad t \in I_{i}, \quad i=1, \ldots, N \\
\mathbf{x}\left(t=T_{i}\right)=\left.\mathbf{x}\right|_{I_{i-1}}\left(t=T_{i}\right), \quad \text { for } \quad i=2, \ldots, N \quad \text { and } \quad \mathbf{x}\left(t=T_{1}\right)=\mathbf{x}_{0} .
\end{array}\right.
$$

The objective of our optimization strategy is to determine an admissible $u(t)$ that ensures the best blood purification. This corresponds to maximize the total amount of extracted toxins:

$$
J(\mathbf{t})=\int_{T_{1}}^{T_{N+1}} \sum_{i=1}^{M} w_{i} x_{i}(t)|\min [u[\mathbf{t}](t), 0]| d t:=\int_{T_{1}}^{T_{N+1}} L(\mathbf{x}, u) d t
$$

where $w_{i}$ are suitable weights associated to the components of $\mathbf{x}$ and the notation $u[\mathbf{t}](t)$ reminds that the control function $u(t)$ is uniquely identified by the control vector $\mathbf{t} \in \mathbb{R}^{\text {dof }}$. The weights $w_{i}$ are positive for the chemical species considered to be toxins while are null otherwise. The quantity $J(\mathbf{t})$ is thus our cost functional. We are now in position to set up our control problem.

Problem 2. Given $N$, find $\mathbf{t}^{*} \in \mathbb{R}^{\text {dof }}$ such that

$$
J\left(\mathbf{t}^{*}\right)=\max _{\mathbf{t} \in \mathcal{T} \subset \mathbb{R}^{\text {dof }}} J(\mathbf{t})
$$


under the constraints:

$$
\begin{gathered}
\dot{\mathbf{x}}=f(\mathbf{x}, u), t \in I_{i} \text { with } \mathbf{x}\left(T_{i}\right)=\left.\mathbf{x}\right|_{I_{i-1}}\left(T_{i}\right), \text { for } i=2, \ldots, N \text { and } \mathbf{x}\left(T_{1}\right)=\mathbf{x}_{0} \\
\mathcal{T}=\left\{\mathbf{t} \in \mathbb{R}^{\text {dof }} \mid g_{i}(\mathbf{t}) \leq 0 \text { with } i=1, \ldots, N-1 \text { and } h(\mathbf{t})=0\right\}
\end{gathered}
$$

We notice that the number of cycles is a fixed parameter in our setting. However, a reduction of the number of cycles can be achieved by collapsing a cycle (e.g. the $i^{\text {th }}$ cycle), that corresponds to overlay the switching times $t_{i} \in\left[T_{i}, T_{i+1}\right]$. Conversely, the optimization algorithm that we will set up will not be able to increase the number of cycles. For the specific application of PD, this is not a restriction because clinical evidence and theoretical observations show that the optimal choice of $N$ is the minimum integer that allows to exploit the available dialyzing fluid.

\section{The optimal control strategy}

Our aim is twofold: first of all we would like to build up an optimization algorithm that picks up the best dialysis profile for a specific patient; the second step will be to develop a mathematical method that allows to compare the efficacy of the different ways of performing PD which are established in clinical practice (see [11] for a review on this subject). Indeed, this study would be very useful in providing a rationale to put into evidence the general characteristics of the most efficient therapy. The key point that has to be considered in both cases is the set up of a procedure to compute the gradient of the cost functional $J(\mathbf{t})$ with respect to the vector of degrees of freedom t. In what follows, we describe in detail this procedure, which stands on classical results of optimal control theory.

First of all, let us introduce a fictitious time $\tau$ and a piecewise linear map from $\tau$ to $t$, that is denoted with $t=\mathcal{M}[\mathbf{t}](\tau), \mathcal{M}[\mathbf{t}]:[1$, dof +1$] \rightarrow\left[T_{1}, T_{N+1}\right]$ such that $\mathcal{M}[\mathbf{t}](\tau)=t_{i}+\left(t_{i+1}-t_{i}\right)(\tau-i)$. This map satisfies, $\mathcal{M}[\mathbf{t}](\tau=i)=t_{i}$ for $i=1, \ldots$, dof +1 and this shows that the control $u(t)$ can be expressed as the transformation of a fixed function $\bar{u}(\tau)$ (we will denote with $\bar{v}$ the functions depending on the fictitious time $\tau)$. More precisely, $u(t)=\bar{u}\left(\mathcal{M}^{-1}[\mathbf{t}](t)\right)$ where $\bar{u}(\tau)$ is defined as follows,

$$
\bar{u}(\tau)=\left\{\begin{array}{l}
0, \tau \in[2 i-1,2 i) \\
-U, \tau \in[2 i, 2 i+1 / 2) \\
+U, \tau \in[2 i+1 / 2,2 i+1)
\end{array} \quad i=1, \ldots, N\right.
$$

Secondly, we introduce the auxiliary state variables $\bar{x}_{M+i}(\tau), i=1, \ldots$, dof that satisfy the following auxiliary state equations for $\tau \in[1, \operatorname{dof}+1]$,

$$
\left\{\begin{array}{l}
\dot{\bar{x}}_{M+i}(\tau)=0 \\
\bar{x}_{M+i}(1)=t_{i}
\end{array} \Rightarrow \bar{x}_{M+i}(\tau)=t_{i}, \tau \in[1, \operatorname{dof}+1], i=1, \ldots,\right. \text { dof }
$$

We denote with $\overline{\mathbf{X}}=\left[\bar{x}_{1}, \ldots, \bar{x}_{M}, \bar{x}_{M+1}, \ldots, \bar{x}_{M+\text { dof }}\right]^{T}$ the new state vector and with $\overline{\mathbf{F}}=\left[f_{1}, \ldots, f_{M}, \mathbf{0}\right]^{T}$ the right hand side of the state equation modified according to (2.1). As a consequence of that, the vector function $\overline{\mathbf{X}}(\tau)$ satisfies, 
Problem 3. Given $\mathbf{X}_{0}$, find $\mathbf{X}(\tau) \in\left(C^{0}([1, \operatorname{dof}+1])\right)^{M+\text { dof }}$ such that,

$$
\left\{\begin{array}{l}
\dot{\overline{\mathbf{X}}}(\tau)=\dot{\mathcal{M}}[\mathbf{t}](\tau) \bar{F}(\overline{\mathbf{X}}, u), \tau \in \bar{I}_{i}=(i, i+1), i=1, \ldots, N \\
\overline{\mathbf{X}}(\tau=i)=\left.\overline{\mathbf{X}}\right|_{\bar{I}_{i-1}}(\tau=i), i=2, \ldots, N \text { and } \mathbf{\mathbf { X }}(\tau=1)=\mathbf{X}_{0}
\end{array}\right.
$$

By virtue of the identification between the degrees of freedom $t_{i}$ and the auxiliary state variables $\bar{x}_{M+i}$ we can write $\mathcal{M}[\mathbf{t}](\tau)=\mathcal{M}\left[\bar{x}_{M+i}\right](\tau)$. We observe that the degrees of freedom of the control now appear as initial conditions of the state vector and this will be very useful in order to derive a computable expression of the gradient of $J(\mathbf{t})$ with respect to the degrees of freedom $\mathbf{t}$, under the constraint represented by the state equation. Let us now establish a necessary condition that characterizes the optimal control function $u\left[\mathbf{t}^{*}\right](t)$. By applying the optimal control theory, and in particular the necessary conditions for Lagrange and Bolza problems (see for example [1], Ch.5, Th.5.1), we observe that the optimal control vector $\mathbf{t}^{*}$ satisfies the following property.

Property 1. Given the augmented functional $J_{A}(\mathbf{t})$,

$$
J_{A}(\mathbf{t})=\sum_{i=1}^{\text {dof }} \int_{i}^{i+1}\left\{L(\overline{\mathbf{X}}, \bar{u}) \dot{\mathcal{M}}\left[\bar{x}_{M+i}\right]+\bar{\lambda}^{T}\left(\dot{\mathcal{M}}\left[\bar{x}_{M+i}\right] \bar{F}(\overline{\mathbf{X}}, \bar{u})-\dot{\overline{\mathbf{X}}}\right)\right\} d \tau
$$

there exists a dynamic multiplier $\lambda(\tau) \in\left(C^{0}(1 \text {, dof }+1)\right)^{M+\text { dof }}$ such that $\nabla_{\mathbf{t}} J_{A}\left(\mathbf{t}^{*}\right)=0$, where $\lambda(\tau)$ is the backward solution of the following system of equations,

$$
\left\{\begin{array}{l}
\dot{\bar{\lambda}}=-\nabla_{\overline{\mathbf{X}}} H(\overline{\mathbf{X}}, \bar{u}, \bar{\lambda}), \tau \in \bar{I}_{i}=(i, i+1) \\
\bar{\lambda}(\tau=i+1)=\left.\bar{\lambda}\right|_{\bar{I}_{i+1}}(\tau=i+1) \text { for } i=\operatorname{dof}-1, \ldots, 1 \text { and } \bar{\lambda}(\operatorname{dof}+1)=0
\end{array}\right.
$$

being $H(\overline{\mathbf{X}}, \bar{u}, \bar{\lambda})$ the Hamiltonian function associated to our control problem, precisely,

$$
H(\overline{\mathbf{X}}, \bar{u}, \bar{\lambda})=\dot{\mathcal{M}}\left[\bar{x}_{M+i}\right]\left\{L(\overline{\mathbf{X}}, \bar{u})+\bar{\lambda}^{T} \bar{F}(\overline{\mathbf{X}}, \bar{u})\right\}
$$

A consequence of property 1 is that the optimal control $\mathbf{t}^{*}$ lays in the set of the stationary points of $J_{A}$. Consequently, we aim to set up a mathematical method that identifies the vectors $\mathbf{t}$ such that $\nabla_{\mathbf{t}} J_{A}\left(\mathbf{t}^{*}\right)=0$. In absence of more restrictive or sufficient conditions for optimality, we will identify $\mathbf{t}^{*}$ with the maximizer of $J_{A}(\mathbf{t})$. In order to derive a computable expression of $\nabla_{\mathbf{t}} J_{A}(\mathbf{t})$ we observe that, by means of standard integration by parts into (2.3) we obtain,

$$
\begin{aligned}
J_{A}(\mathbf{t})=\sum_{i=1}^{\text {dof }} \int_{i}^{i+1}\left\{\dot{\mathcal{M}}\left[\bar{x}_{M+i}\right] L(\overline{\mathbf{X}}, \bar{u})+\dot{\mathcal{M}}\left[\bar{x}_{M+i}\right] \bar{\lambda}^{T} \bar{F}(\overline{\mathbf{X}}, \bar{u})\right. & \left.\left.+\dot{\bar{\lambda}}^{T} \overline{\mathbf{X}}\right)\right\} d \tau \\
& +\sum_{i=1}^{\operatorname{dof}}\left\{\bar{\lambda}^{T}(i) \overline{\mathbf{X}}(i)-\bar{\lambda}^{T}(i+1) \overline{\mathbf{X}}(i+1)\right\}
\end{aligned}
$$

Let now be $d t_{i}, i=1, \ldots$, dof an infinitesimal perturbation on the control parameters and let $d \bar{X}_{j}(\tau), j=1, \ldots, M+$ dof be the corresponding perturbation on $\overline{\mathbf{X}}$. We observe that the resulting perturbation on $J_{A}$ is,

$$
d J_{A}=\sum_{i=1}^{\text {dof }} \int_{i}^{i+1}\left\{\nabla_{\overline{\mathbf{X}}} H(\overline{\mathbf{X}}, \bar{u}, \bar{\lambda})+\dot{\bar{\lambda}}^{T}(\tau)\right\} d \overline{\mathbf{X}}(\tau) d \tau+\sum_{i=1}^{\text {dof }}\left\{\bar{\lambda}^{T}(i) d \overline{\mathbf{X}}(i)-\bar{\lambda}^{T}(i+1) d \overline{\mathbf{X}}(i+1)\right\}
$$


and by substituting (2.4) into (2.5) we obtain $d J_{A}=\bar{\lambda}^{T}(\tau=1) d \overline{\mathbf{X}}(\tau=1)$. In order to determine $\nabla_{\mathbf{t}} J_{A}(\mathbf{t})$ we remind that $d \bar{X}_{j}(\tau=1)=0, j=1, \ldots, M$ (because the initial conditions of the physical state variables in (1.8) are fixed and thus independent from the vector $\mathbf{t}$ ) and that $d \bar{X}_{j}(\tau=1)=d t_{i}$, $j=M+i, i=1, \ldots$, dof (according to the definition of the auxiliary state variables in (2.1)). By replacing the previous expressions into (2.5) we finally obtain,

$$
d J_{A}=\bar{\lambda}^{T}(\tau=1) d \overline{\mathbf{X}}(\tau=1)=\sum_{i=1}^{\text {dof }} \bar{\lambda}_{M+i}(\tau=1) d t_{i},
$$

that is

$$
\frac{\partial J_{A}}{\partial t_{i}}=\bar{\lambda}_{M+i}(\tau=1), i=1, \ldots, \text { dof } .
$$

This result will be applied in the next section in order to set up a maximization algorithm of the functional $J_{A}(\mathbf{t})$.

\section{Numerical algorithms}

Let us consider the original formulation of our control problem, that is problem 2. We observe that the determination of the optimal function $u\left[\mathbf{t}^{*}\right](t)$ is restricted by two types of constraint: a dynamic constraint represented by equation (1.8) and some inequality and equality constraints $g_{i}(\mathbf{t}) \leq 0, h(\mathbf{t})=0$ defined in (1.6) and (1.7) respectively, which represent a restriction of the space of the admissible controls, $\mathcal{T}$. On one hand, by means of property 1 it has been possible to take into account of the dynamic constraint, with the introduction of the augmented functional $J_{A}(\mathbf{t})$ and of the dynamic multiplier $\bar{\lambda}(\tau)$. On the other hand, the problem of enforcing the algebraic constraints (1.6) and (1.7) is a classical issue in the theory of constrained optimization. Indeed many general algorithms has been developed in order to treat such problems, for instance we mention sequential quadratic programming and augmented Lagrangian methods (see for example [10] for an overview). According to the fact that we have already applied an augmented Lagrangian approach for the treatment of dynamic constraint set by (1.8), we apply the augmented Lagrangian method (see [14] and in particular [2]) in order to consider the algebraic constraints (1.6) and (1.7). Here, we would like to describe the main steps of the procedure that we have set up. According to property 1 , our original control problem has been reformulated as the maximization of $J_{A}(\mathbf{t})$ defined in (2.3) under algebraic constraints. Starting from this basis, we consider the following basic maximization algorithm inspired to the augmented Lagrangian method.

Algorithm 1. Given $q^{(0)}, p_{i}^{(0)}, i=1, \ldots, N-1$ and $c \geq 0$, for $k=1,2,3, \ldots$ find $\mathbf{t}^{(k)}$ such that,

$$
J_{A A}(\mathbf{t}(k))=\max _{\mathbf{t} \in \mathcal{T}} J_{A A}(\mathbf{t})
$$

where,

$$
\begin{gathered}
J_{A A}(\mathbf{t})=J_{A}(\mathbf{t})+q^{(k)} h(\mathbf{t})+\sum_{i=1}^{N-1} p_{i}^{(k)} \gamma_{i}^{(k)}(\mathbf{t})+r^{(k)}\left(h^{2}(\mathbf{t})+\sum_{i=1}^{N-1}\left(\gamma_{i}^{(k)}(\mathbf{t})\right)^{2}\right) \\
q^{(k+1)}=q^{(k)}+2 r^{(k)} h\left(\mathbf{t}^{(k)}\right), p_{i}^{(k+1)}=p_{i}^{(k)}+2 r^{(k)} \gamma_{i}^{(k)}\left(\mathbf{t}^{k}\right), r^{(k+1)}=c r^{(k)} \\
\gamma_{i}^{(k)}=\max \left[g_{i}(\mathbf{t}) ;-\frac{p_{i}^{(k)}}{2 r^{(k)}}\right]
\end{gathered}
$$


For the initialization of the algorithm we assume to start from an admissible point $\mathbf{t}^{(0)}$ internal to $\mathcal{T}$ and according to that we chose $p_{i}^{(0)}=0$ and $q^{(0)}$ as the projection of $\nabla_{\mathbf{t}} J_{A}\left(\mathbf{t}^{(0)}\right)$ along the direction normal to $h(\mathbf{t})$ in the point $\mathbf{t}^{(0)}$, namely,

$$
q^{(0)}=-\frac{\nabla_{\mathbf{t}} J_{A}\left(\mathbf{t}^{0}\right) \cdot \nabla_{\mathbf{t}} h\left(\mathbf{t}^{0}\right)}{\left\|\nabla_{\mathbf{t}} h\left(\mathbf{t}^{0}\right)\right\|_{2}} .
$$

This expression can be calculated by computing the gradient $\nabla_{\mathbf{t}} h\left(\mathbf{t}^{(0)}\right)$ and by applying (2.6) to approximate $\nabla_{\mathbf{t}} J_{A}\left(\mathbf{t}^{(0)}\right)$ with numerical techniques. Secondly, to solve the maximization problem that arises at each iteration, we consider the line search method (see for example $[4,9,10]$ ), because it exploits the information on the gradient of the functional to maximize. This information is available in our case since $\nabla_{\mathbf{t}} h(\mathbf{t})$ and $\nabla_{\mathbf{t}} g_{i}(\mathbf{t}), i=1, \ldots, N-1$ are easily calculated, while $\nabla_{\mathbf{t}} J_{A}(\mathbf{t})$ can be numerically approximated exploiting (2.6). Efficient implementations of the line search method are available in the Matlab optimization toolbox. To apply such algorithm it is necessary to compute the functional $J_{A A}(\mathbf{t})$ and its gradient $\nabla_{\mathbf{t}} J_{A A}(\mathbf{t})$ at a given point $\mathbf{t}$. We observe that,

$$
\nabla_{\mathbf{t}} J_{A A}(\mathbf{t})=\nabla_{\mathbf{t}} J_{A}(\mathbf{t})+q^{(k)} \nabla_{\mathbf{t}} h(\mathbf{t})+\sum_{i=1}^{N-1} p_{i}^{(k)} \nabla_{\mathbf{t}} \gamma_{i}^{(k)}(\mathbf{t})+2 r^{(k)} h(\mathbf{t}) \nabla_{\mathbf{t}} h(\mathbf{t})+2 r^{(k)} \sum_{i=1}^{N-1} \gamma_{i}^{(k)}(\mathbf{t}) \nabla_{\mathbf{t}} \gamma_{i}^{(k)}(\mathbf{t})
$$

The key point is the approximation of $\nabla_{\mathbf{t}} J_{A}(\mathbf{t})$ with numerical techniques. The main steps of this method are reported below.

Algorithm 2. Given $\mathbf{t} \in \mathbb{R}^{\text {dof }}$ :

compute $\overline{\mathbf{X}}_{h}$, the numerical solution of (2.2);

compute $\bar{\lambda}_{h}$, the numerical solution of (2.4), where we have replaced $\overline{\mathbf{X}}_{\text {with }} \overline{\mathbf{X}}_{h}$ into $\nabla_{\overline{\mathbf{X}}} H(\overline{\mathbf{X}}, \bar{u}, \bar{\lambda})$;

compute $\frac{\partial J_{A}}{\partial t_{i}}=\bar{\lambda}_{h, M+i}(\tau=1), i=1, \ldots$, dof.

For the numerical solution of the system of ordinary differential equations we apply high order, adaptive Runge-Kutta methods. Precisely, we consider the so called DOPRI5 method (see [5]) implemented in Matlab in the subroutine ole45. In the following section we will apply the numerical methods presented for the optimization of PD therapy profile to realistic clinical cases.

\section{Applications}

In this section we discuss the application to PD of the optimization strategy that we have set up. As already mentioned, we address several objectives. First of all, we aim to apply our optimization strategy in order to identify, for a specific patient, the sequence of cycles that maximize the extraction of toxins. Let us consider the following data that characterize the PD therapy that patients with renal disease receive daily. The duration of the therapy is fixed to $T_{t o t}=12$ hours (720 minutes), the available quantity of dialayzing fluid is $V_{t o t}=8$ liter and the maximal volume that can be injected into the peritoneal cavity is $V_{\max }=2$. Starting from these data we pick up randomly one therapy in the set of the admissible ones. This therapy is represented in figure 2 
(left) in terms of volume of dialayzing fluid, $V(t)$, that is present at each time in the peritoneal cavity. We observe that $V(t):=\int_{T_{1}}^{t} u(s) d s$ with $t \in\left(T_{1}, T_{N+1}\right]$ is the integral function of the pump flow rate. Furthermore we consider realistic physiological data for the definition of the parameters that appear in the state equation (1.3). These parameters are reported and determined on the basis of clinical measurements in [6]. We apply our optimization algorithm starting from the therapy described in figure 2 on the left, and on the right we show the profile of $V(t)$ corresponding to the optimal solution obtained when the algorithm has converged satisfying a tolerance equal to $10^{-2}$ on the relative incremental of $\mathbf{t}$. The cost functional, which is defined as the amount of urea extracted during the therapy, has increased from 10.21 grams to 11.30 grams which corresponds to a sensible improvement of $10.12 \%$. A similar optimal solution is obtained starting from different initial guesses of the therapy profile. This observation confirms that our algorithm is well suited for the identification of the optimal therapy for a specific patient. Furthermore, we observe that the optimal solution exploits the maximal admissible volume, $V_{\max }$, at each cycle. As a consequence of that the optimal therapy features the minimal number of cycles that is sufficient to exploit the available total volume of dialayzing fluid, which is equal to the smallest $N \in \mathbb{N}$ such that $N \geq V_{\text {tot }} / V_{\text {max }}$. On the other hand the duration of each cycle is not uniform.
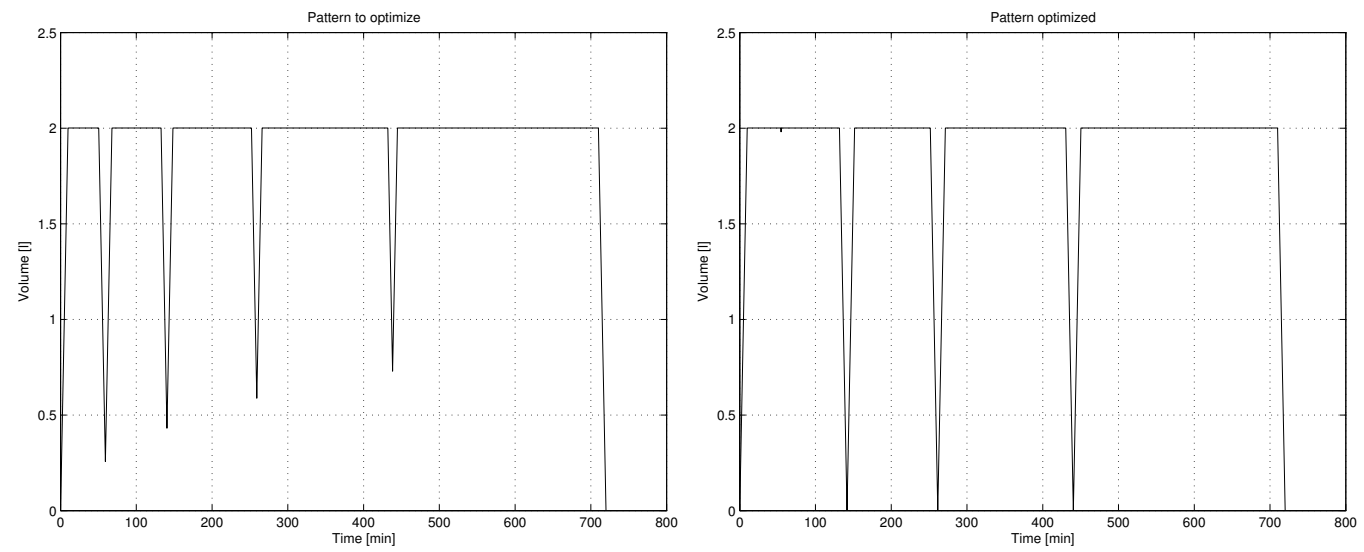

Figure 2: Initial (left) and final (right) therapy profiles in the test of the optimization strategy.

Actually, we notice that the standard PD therapy, which is characterized by cycles that exploit the maximal volume, $V_{\max }$, and of uniform period, equal to $T_{t o t} / N$, turns out to be very similar the optimal one. A slight margin of improvement can be obtained by tuning the period of the cycles. This observation is confirmed by the analysis of the gradient $\nabla_{\mathbf{t}} J_{A}\left(\mathbf{t}^{+}\right)$, where $\mathbf{t}^{+}$is the set of control parameters that characterize the standard PD therapy. For this study, we consider the following data: $V_{t o t}=10$ liter, $V_{\max }=2$ liter, $T_{t o t}=7$ hour and $N=5$. In table 1 we show the gradient of $J_{A}$ with respect to the instants $t_{j} j=1, \ldots, 3(N-1)$ that identify the standard dialysis profile, which are also put into evidence in the scheme of table 1 . We observe that the gradient $\nabla_{\mathbf{t}} J_{A}\left(\mathbf{t}^{+}\right)$can be split in two components, denoted with $\nabla_{\mathbf{t}} J_{A, 1}\left(\mathbf{t}^{+}\right)$and $\nabla_{\mathbf{t}} J_{A, 2}\left(\mathbf{t}^{+}\right)$such that $\nabla_{\mathbf{t}} J_{A}\left(\mathbf{t}^{+}\right)=\nabla_{\mathbf{t}} J_{A, 1}\left(\mathbf{t}^{+}\right)+\nabla_{\mathbf{t}} J_{A, 2}\left(\mathbf{t}^{+}\right)$and that are reported in table 1. The first one, namely $\nabla_{\mathbf{t}} J_{A, 1}\left(\mathbf{t}^{+}\right)$, puts into evidence that it is possible to improve the cost functional by considering a variable cycle period. In particular, since the gradient is positive, the cycles at the beginning of the therapy should be longer than the ones at the end of it. The second component of the gradient, 
namely $\nabla_{\mathbf{t}} J_{A, 2}\left(\mathbf{t}^{+}\right)$, shows that an increment of the cost functional can be achieved by increasing the volume that is exchanged at each cycle. Moreover, we observe that the components of $\nabla_{\mathbf{t}} J_{A, 2}\left(\mathbf{t}^{+}\right)$ are almost double (in absolute value) with respect to the ones of $\nabla_{\mathbf{t}} J_{A, 1}\left(\mathbf{t}^{+}\right)$. This means that the cost functional $J_{A}$ is more sensitive to variations in the volume exchanged at each cycle than to variations of the period of the cycle. However, for the specific therapy at hand, the inequality constraints (1.4) do not allow to further increase the volume exchanged at each cycle because the maximal peritoneal capacity, $V_{\max }$ can not be exceeded. In other words, the component $\nabla_{\mathbf{t}} J_{A, 2}\left(\mathbf{t}^{+}\right)$ can not be exploited for the improvement of $J_{A}$. To sum up, this analysis shows that the standard way to perform PD therapy (repeated cycles with similar characteristics) is quasi optimal, in the sense that it provides the optimal repartition of the dialyzing fluid among cycles but it can be enhanced by adjusting the repartition among the cycles of the available time for the therapy.

Table 1: The gradient of $J_{A}$ with respect to the instants $t_{j}, j=1, \ldots, 3(N-1)$

\begin{tabular}{|c|c|c|c|c|c|c|}
\hline$V(t) \uparrow$ & $\stackrel{\mathrm{t} 1}{\leftrightarrow}$ & $t^{3}=t^{t 4}$ & $\stackrel{t 6}{\longrightarrow}$ & t9 & $\stackrel{\mathrm{t} 12}{\rightleftharpoons}$ & \\
\hline$\frac{d J_{A}}{d t_{i}}$ & $\begin{array}{c}t_{1} \\
-0.0023 \\
\end{array}$ & $\begin{array}{c}t_{2} \\
0.0023\end{array}$ & $\begin{array}{c}t_{3} \\
0.0069 \\
\end{array}$ & $\begin{array}{c}t_{4} \\
-0.0024 \\
\end{array}$ & $\begin{array}{c}t_{5} \\
0.0024 \\
\end{array}$ & $\begin{array}{c}t_{6} \\
0.0074 \\
\end{array}$ \\
\hline$\frac{d J_{A}}{d t_{i}}$ & $\begin{array}{c}t_{7} \\
-0.0025 \\
\end{array}$ & $\begin{array}{c}t_{8} \\
0.0025\end{array}$ & $\begin{array}{c}t_{9} \\
0.0077 \\
\end{array}$ & $\begin{array}{c}t_{10} \\
-0.0026\end{array}$ & $\begin{array}{c}t_{11} \\
0.0026 \\
\end{array}$ & $\begin{array}{c}t_{12} \\
0.0079 \\
\end{array}$ \\
\hline$\frac{d J_{A, 1}}{d t_{i}}$ & $\begin{array}{c}t_{1} \\
0.0023 \\
\end{array}$ & $\begin{array}{c}t_{2} \\
0.0023 \\
\end{array}$ & $\begin{array}{c}t_{3} \\
0.0023 \\
\end{array}$ & $\begin{array}{c}t_{4} \\
0.0024 \\
\end{array}$ & $\begin{array}{c}t_{5} \\
0.0024 \\
\end{array}$ & $\begin{array}{c}t_{6} \\
0.0024 \\
\end{array}$ \\
\hline$\frac{d J_{A, 1}}{d t_{i}}$ & $\begin{array}{c}t_{7} \\
0.0025 \\
\end{array}$ & $\begin{array}{c}t_{8} \\
0.0025 \\
\end{array}$ & $\begin{array}{c}t_{9} \\
0.0025 \\
\end{array}$ & $\begin{array}{c}t_{10} \\
0.0026 \\
\end{array}$ & $\begin{array}{c}t_{11} \\
0.0026 \\
\end{array}$ & $\begin{array}{c}t_{12} \\
0.0026 \\
\end{array}$ \\
\hline$\frac{d J_{A, 2}}{d t_{i}}$ & $\begin{array}{c}t_{1} \\
-0.0046 \\
\end{array}$ & $\begin{array}{c}t_{2} \\
0.0 \\
\end{array}$ & $\begin{array}{c}t_{3} \\
0.0046 \\
\end{array}$ & $\begin{array}{c}t_{4} \\
-0.0048 \\
\end{array}$ & $\begin{array}{c}t_{5} \\
0.0 \\
\end{array}$ & $\begin{array}{c}t_{6} \\
0.0050 \\
\end{array}$ \\
\hline$\frac{d J_{A, 2}}{d t_{i}}$ & $\begin{array}{c}t_{7} \\
-0.0050\end{array}$ & $\begin{array}{c}t_{8} \\
0.0\end{array}$ & $\begin{array}{c}t_{9} \\
0.0052\end{array}$ & $\begin{array}{c}t_{10} \\
-0.0052\end{array}$ & $\begin{array}{l}t_{11} \\
0.0\end{array}$ & $\begin{array}{c}t_{12} \\
0.0053\end{array}$ \\
\hline
\end{tabular}

\section{References}

[1] L. Cesari. Optimization - theory and applications, volume 17 of Applications of Mathematics (New York). Springer-Verlag, New York, 1983. Problems with ordinary differential equations.

[2] Andrew R. Conn, Nicholas I. M. Gould, and Philippe L. Toint. A globally convergent augmented Lagrangian algorithm for optimization with general constraints and simple bounds. SIAM J. Numer. Anal., 28(2):545-572, 1991.

[3] F.R.E. Curry. Handbook of Physiology, chapter 8: Mechanics and thermodynamics of transcapillary exchange. E.M. Renkin ed., American Physiological Society, Bethesda, Maryland, 1984. 
[4] R. Fletcher. Practical methods of optimization. A Wiley-Interscience Publication. John Wiley \& Sons Ltd., Chichester, second edition, 1987.

[5] E. Hairer, S. P. Nørsett, and G. Wanner. Solving ordinary differential equations. I, volume 8 of Springer Series in Computational Mathematics. Springer-Verlag, Berlin, second edition, 1993. Nonstiff problems.

[6] B. Haraldsson. Assessing the peritoneal dialysis capacities of individual patients. Kidney Int., 47:1187-1198, 1995.

[7] A. Katchalsky and P.F. Curran. Nonequilibrium Thermodynamics in Biophysics. Harvard University Press, 1981.

[8] O. Kedem and A. Katchalsky. Thermodynamic analysis of the permeability of biological membranes to non-electrolytes. Biochimica et Biophysica Acta, pages 229-246, 1958.

[9] C. T. Kelley. Iterative methods for optimization, volume 18 of Frontiers in Applied Mathematics. Society for Industrial and Applied Mathematics (SIAM), Philadelphia, PA, 1999.

[10] J. Nocedal and S.J. Wright. Numerical Optimization. Springer Verlag, New York, 1999.

[11] K.D. Nolph, editor. Peritoneal Dialysis. Nijhoff, Boston, 1981.

[12] W.K. Pyle. Mass transfer in peritoneal dialysis. PhD thesis, University of Texas, Ausin, 1981.

[13] B. Rippe. A three-pore model of peritoneal transport. Perit. Dial. Int., 13:35-38, 1992.

[14] R. T. Rockafellar. Lagrange multipliers and optimality. SIAM Rev., 35(2):183-238, 1993.

[15] E.F. Vonesh, M.J. Lysaght, J. Moran, and P. Farrell. Kinetic modeling as a prescription aid in peritoneal dialysis. Blood Purif., 9:246-270, 1991. 